\title{
PRIMEROS DATOS SOBRE EL TRANSPORTE, PROCESAMIENTO Y CONSUMO DE GUANACOS EN LA LOCALIDAD ARQUEOLÓGICA HESHKAIA (SUDESTE DE TIERRA DEL FUEGO, ARGENTINA)
}

\author{
DANIELA ALUNNI" Y ATILIO FRANCISCO ZANGRANDO"
}

\begin{abstract}
RESUMEN
Se presentan los resultados del análisis faunístico efectuado para la localidad arqueológica Heshkaia (Moat, sudeste de Tierra del Fuego), cuyas ocupaciones registran edades radiocarbónicas comprendidas entre ca. 1.600-200 años AP. Las excavaciones y estudios efectuados hasta el momento señalan que los guanacos (Lama guanicoe) era el principal recurso explotado en este sector. Aquí centramos la atención sobre el procesamiento y consumo de este ungulado, contemplando también los procesos tafonómicos que afectaron las muestras. Moat ha sido un espacio arqueológicamente poco explorado, por lo que estos datos suman importancia desde una perspectiva regional para un mayor entendimiento de las actividades de subsistencia por parte de las poblaciones que habitaron la costa sur de Tierra del Fuego durante el Holoceno Tardío.
\end{abstract}

PALABRAS CLAVE: Moat, cazadores-recolectores, zooarqueología, Holoceno Tardío.

\section{FIRST DATA OF GUANACO TRANSPORT, BUTCHERING AND CONSUMPTION AT HESHKAIA ARCHAEOLOGICAL LOCALITY (SOUTHEASTERN OF TIERRA DEL FUEGO, ARGENTINA)}

\section{ABSTRACT}

We present the results of faunal studies conducted on the Heshkaia archaeological locality (Moat, southeastern of Tierra del Fuego), which occupations span from ca. 1.600 to 200 radiocarbon years BP. At the moment, the excavations and studies carried out indicate that guanacos (Lama guanicoe) were the main resources exploited in this sector of Tierra del Fuego. In this paper, we focus on processing and consumption patterns of this ungulate, and we also consider taphonomic processes that could affect the samples. These data allow a better understanding of the subsistence activities of the populations which inhabited the southern coast of Tierra del Fuego during the Late Holocene.

KEYWORDS: Moat, hunter-gatherers, zooarchaeology, Late Holocene.

Asociación de Investigaciones Antropológicas, Facultad de Filosofía y Letras, Universidad de Buenos Aires. E-mail: alunni_d@ yahoo.com.ar.

*. CONICET-CADIC, Universidad de Buenos Aires. E-mail: panchozan@yahoo.com.ar, afzangrando@cadic-conicet.gob.ar. 


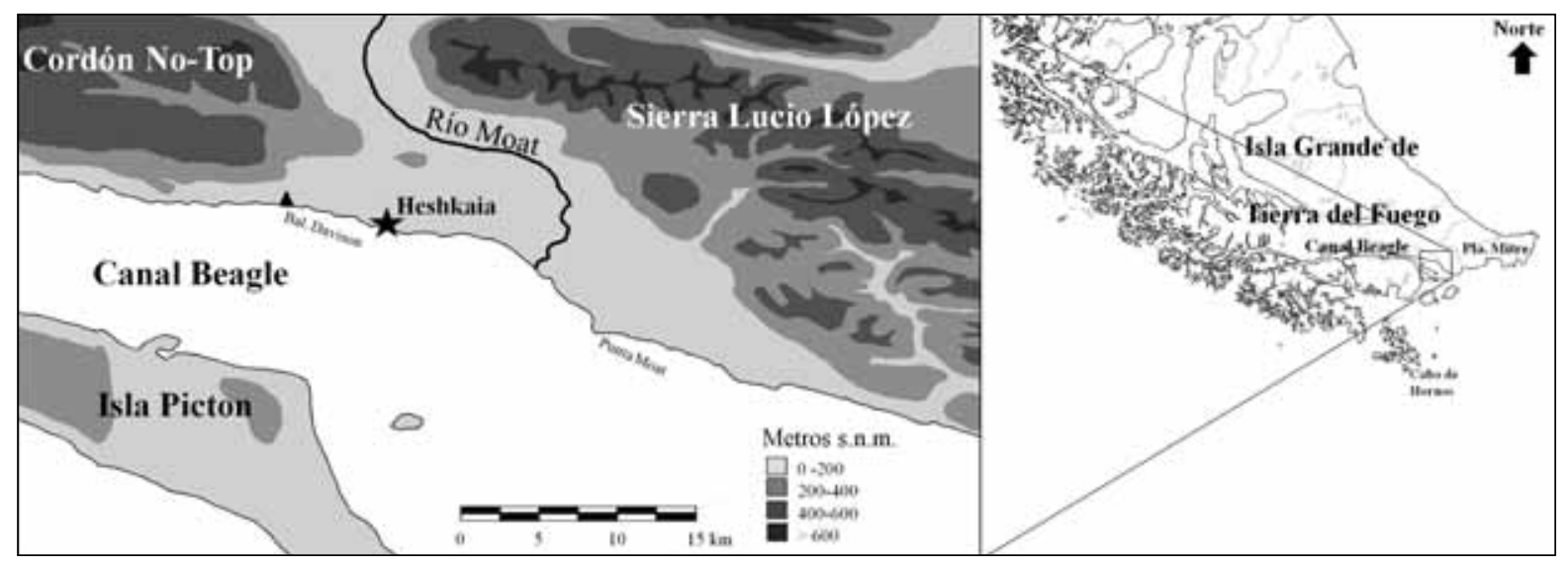

Fig. 1. Cuenca del río Moat y localidad arqueológica Heshkaia.

\section{INTRODUCCIÓN}

Recientemente se ha dado comienzo a una exploración sistemática del registro arqueológico en el valle del río Moat (Figura 1): este espacio comprende toda la cuenca del río homónimo y el tramo de costa que se extiende desde baliza Davison (5456'33.31" S -6654'13.80" O) hasta cabo San Pío (55 3'19.13" S - 66²7’50.42” O). Este sector se integra dentro de las discusiones de un proyecto marco que además incluye otras dos unidades contiguas al paisaje de Moat: el cordón No-Top hacia el oeste y la bahía Sloggett hacia este (Zangrando 2010). Es un ámbito en el cual no se han desarrollado investigaciones arqueológicas previas, por consiguiente como primer objetivo se buscó analizar el paisaje arqueológico, explorar la composición de los conjuntos arqueológicos y ofrecer información cronológica para poder evaluar la utilización del espacio y de sus recursos por parte de las poblaciones cazadoras-recolectoras (Zangrando 2010). Las prospecciones identificaron hasta el momento 41 sitios distribuidos en seis localidades arqueológicas, de las cuales se escogió Heshkaia para realizar las primeras exploraciones por evidenciar una alta intensidad de uso humano y diversidad en cuanto a la forma de sitios, representando un caso apropiado para abordar el objetivo planteado (Zangrando et al. 2011).

La localidad Heshkaia se encuentra en el tramo costero central de Moat (Figura 1), por donde tiene su curso el río homónimo. Como veremos a continuación, la cuenca de este río se destaca como rasgo geográfico y ecológico. Sobre el total de 13 sitios registrados en esta localidad, los sondeos y excavaciones se llevaron a cabo en cinco de ellos con ubicación, tamaños y aspectos superficiales disímiles (Tabla 1).

A partir de los materiales recuperados se obtuvieron algunos resultados arqueológicos interesantes relacionados con la subsistencia de los grupos cazadores-recolectores que habitaron ese espacio. La elevada densidad de sitios hallada en esta localidad arqueológica parece señalar un uso

Tabla 1. Principales características de los sitios.

\begin{tabular}{|c|c|c|c|c|c|c|}
\hline Sitios & Aspecto superficial & $\begin{array}{c}\text { Cronología } \\
\text { (C14 AP) }\end{array}$ & Extensión & $\begin{array}{c}\text { Distancia a } \\
\text { la costa }\end{array}$ & $\begin{array}{c}\text { Extensión } \\
\text { del sondeo }\end{array}$ & $\begin{array}{c}\text { Profundidad } \\
\text { máxima }\end{array}$ \\
\hline Heshkaia 28 & Conchero, montículo aislado & $678 \pm 38$ & $20 \mathrm{~m}^{2}$ & $22 \mathrm{~m}$ & $1 \times 1 \mathrm{~m}$ & $29 \mathrm{~cm}$ \\
\hline Heshkaia 30 & $\begin{array}{c}\text { Conchero, tres } \\
\text { montículos alineados }\end{array}$ & $263 \pm 35$ & $80 \mathrm{~m}^{2}$ & $1 \mathrm{~m}$ & $1 \times 1 \mathrm{~m}$ & $75 \mathrm{~cm}$ \\
\hline Heshkaia 34 & $\begin{array}{c}\text { Conchero, cinco montículos } \\
\text { y tres estructuras anulares }\end{array}$ & $981 \pm 36$ & $1500 \mathrm{~m}^{2}$ & $40 \mathrm{~m}$ & $1 \times 1 \mathrm{~m}$ & $63 \mathrm{~cm}$ \\
\hline Heshkaia 35 & Conchero, montículo aislado & $656 \pm 35$ & $40 \mathrm{~m}^{2}$ & $200 \mathrm{~m}$ & $6 \times 1 \mathrm{~m}$ & $58 \mathrm{~cm}$ \\
\hline Heshkaia 39 & Sitio sub-superficial & - & $10 \mathrm{~m}^{2}$ & $300 \mathrm{~m}$ & $2 \times 1 \mathrm{~m}$ & $10 \mathrm{~cm}$ \\
\hline
\end{tabular}


Tabla 2. Representación faunística en la localidad arqueológica Heshkaia en NISP.

\begin{tabular}{|c|c|c|c|c|c|c|c|c|}
\hline Sitios & Pinnípedos & Guanacos & Cánidos & Aves & Peces & Cetáceos & Indeterminados & Totales \\
\hline 28 & 2 & 11 & 0 & 74 & 18 & 5 & 21 & 131 \\
\hline 30 & 21 & 25 & 1 & 444 & 677 & 36 & 59 & 1263 \\
\hline 34 & 62 & 613 & 3 & 90 & 56 & 6 & 555 & 1385 \\
\hline 35 & 6 & 834 & 0 & 144 & 31 & 16 & 374 & 1405 \\
\hline 39 & 7 & 21 & 0 & 6 & 0 & 4 & 19 & 57 \\
\hline Totales & 98 & 1504 & 5 & 758 & 782 & 67 & 1027 & 4241 \\
\hline
\end{tabular}

intensivo y regular de este ámbito costero durante el Holoceno Tardío y la información obtenida hasta el momento sugiere para todos los sitios excavados un mayor uso de mamíferos terrestres que marinos (Tabla 2) (Zangrando 2010; Zangrando et al. 2010). Esta última situación es diferente a la registrada en la costa del canal Beagle (Orquera y Piana 1999a), donde la evidencia arqueológica muestra una mayor importancia de pinnípedos sobre otros recursos.

El objetivo de este informe consiste en evaluar las estrategias de obtención, transporte y consumo de guanacos (Lama guanicoe), ya que éste constituyó la principal presa explotada por los grupos cazadores-recolectores que habitaron Heshkaia. Este análisis complementa y amplía los resultados obtenidos hasta el momento sobre la utilización humana de este espacio, profundizando lo referente a las estrategias de explotación de este ungulado.

\section{CARACTERIZACIÓN DEL AMBIENTE Y DISPONIBILIDAD DE GUANACOS EN MOAT}

El ámbito de Moat se ubica en el tramo oriental de la cordillera Fueguina, caracterizada por serranías $\mathrm{y}$ valles. Las costas tienen pendientes predominantemente suaves. Este sector se caracteriza por una baja altura del espaldar montañoso (las sierras tienen alturas que van de los 100 a $900 \mathrm{~m}$ sobre el nivel del mar), con valles amplios y transversales a la línea de costa, lo que favorece la circulación de guanacos (Raedeke 1976; Montes et al. 2000). Esta situación se intensifica a medida que se acerca el invierno, dado que se realizan desplazamientos desde las zonas altas hacia el litoral debido al impacto de las nevadas, las cuales se manifiestan no sólo por el efecto térmico sobre los individuos, sino también sobre la fuente de alimento disponible (Montes et al. 2000). En estos momentos del año, los guanacos se desplazan siguiendo los cauces de las cuencas de los ríos que desaguan en las costas, dado que en éstas últimas la carga nívea es menor (Fitz-Roy 1839: 186, Martial 1888: 195, Montes et al. 2000, Raedeke 1976, Schiavini et al. 2010, ver también Orquera y Piana 1999b). Por lo tanto, estos lugares constituyen espacios de refugio durante el invierno dado que ofrecen reparo y alimento, por lo que el hallazgo de guanacos es más frecuente durante esta estación. Este patrón de movilidad estacional señalado para guanacos tiene semejanzas temporales con el modelo de explotación de este recurso basado sobre su estructura social propuesto por Lanata (1990) para península Mitre, el cual también plantea que la caza de guanacos era más fructuosa durante el invierno -particularmente sobre grupos familiares.

En Moat existen condiciones naturales que contrastan significativamente con las del canal Beagle central. Un sistema hidrológico diferente combinado con otras condiciones climáticas -mayor precipitación a nivel anual y mayor exposición a los vientos del océano Atlántico Sur (Frangi y Ritcher 1994)- hacen que determinados elementos del paisaje, flora y fauna sean más parecidas a de las condiciones que observamos en península Mitre. El valle de Moat presenta reducidas superficies cubiertas por bosques (en las que se identificaron tres comunidades de Nothofagus) y gramíneas que se restringen a laderas y algunos sectores litorales (Moore 1983). En cambio, se observa un gran desarrollo areal de turbales, que se ubican en zonas aterrazadas y donde se observan turberas de Sphagnum, que son dominantes en el sector occidental (por ejemplo valle Carbajal-Tierra Mayor), y de Astelia comunes en el oriente fueguino (Roig y Collado 2004). También están presentes las denominadas turberas de transición, donde el componente arbóreo es relativamente importante ya que se encuentran entre el ambiente de bosque y el de las turberas antes mencionadas. En estas turberas generalmente están presentes con cobertura variable Marsippospermum grandiflorum (Juncácea), Empetrum rubrum, Astelia pumila 
y Chiliotrichum diffusum, y conjuntamente con los turbales de Juncáceas, tienen una extensión significativamente mayor a las antes mencionadas. Esto no es un aspecto menor, ya que gran parte de su vegetación, y en particular los juncos, es la que registra mayor valor del herbaje junto con los pastizales de gramíneas en el sector de Moat (Haene et al. 2011). El valor pastoril es un indicador adecuado de la calidad forrajera de la vegetación: mientras que bosques y turberas de Astelia tienen valores bajos, los pastizales con arbustos, las praderas con juncos y los juncales mantienen niveles moderadamente elevados en Moat (Haene et al. 2011). Dado que las Juncáceas son un componente importante en la dieta de los guanacos en las zonas de ecotono bosque-estepa (Bonino y Pelliza Sbriller 1991a) y que la dieta de guanacos, ovinos y bovinos en Tierra del Fuego mostró una gran similitud (Bonino y Pelliza Sbriller 1991b), el valor pastoril resulta una medida útil y permite inferir que en Moat se dan buenas condiciones para la alimentación de guanacos, y posiblemente mejores que en situaciones de bosque continuo como las que prevalecieron en la costa central del canal Beagle.

Los estudios sobre distribución, abundancia y comportamiento de guanacos para la costa sur de Tierra del Fuego aún son escasos (Bonino y Fernández 1994; Montes et al. 2000). Por otra parte, no existen estudios de estos mamíferos en turberas, aunque normalmente se observa presencia continua de tropillas de guanacos en estos ambientes de Tierra del Fuego (Schlatter 2004). No obstante, las expectativas para un mayor encuentro de guanacos en Moat pueden ser inferidas a partir del relieve y los mosaicos de vegetación. Por lo tanto, si bien los datos arqueológicos indican que el consumo de guanacos estuvo presente en todo el sur de la porción actualmente argentina de Tierra del Fuego, los factores circunstanciales mencionados generan heterogeneidad en su distribución, lo cual pudo haber condicionado las decisiones de los grupos cazadores-recolectores en cuanto a la explotación de estos ungulados.

\section{METODOLOGÍA}

La muestra total de especímenes de camélidos obtenida hasta ahora en Heshkaia es de 1.504, de los cuales 466 pudieron ser identificados con sus correspondientes unidades anatómicas. Los restos faunísticos proceden de los sondeos previamente mencionados y de una excavación llevada a cabo en Heshkaia 35 realizada por fuera del montículo (ver Tabla 1). Esta última cubrió un área de $5 \mathrm{~m}^{2}$ y alcanzó una profundidad máxima de $20 \mathrm{~cm}$ (Zangrando et al. 2011).

Los restos óseos fueron analizados siguiendo los criterios y la ficha de registro de material faunístico propuestos por G. Mengoni Goñalons (1999). El NISP y MNI fueron las medidas utilizadas para cuantificar la abundancia de camélidos. El primero de ellos se calculó sumando tanto los huesos enteros como los fragmentados, incluyendo también dientes sueltos. El MNI se obtuvo considerando el valor de la unidad anatómica más representada de la muestra, teniendo en cuenta la lateralidad y el estadio de fusión de los especímenes analizados (Mengoni Goñalons 1999; Kaufmann 2009). Asimismo, este último indicador permitió determinar la edad de muerte de los guanacos presentes en los conjuntos.

Para evaluar las pautas de transporte se consideraron la representación y abundancia de partes anatómicas de guanacos medidas en términos de MNE, MAU y MAU\%. El MNE se determinó a partir de las frecuencias de los distintos elementos que conforman la carcasa, considerando las diferentes porciones que se conservan de los huesos largos (proximal, diáfisis y distal), así como las zonas diagnósticas de cada elemento (Mengoni Goñalons 1999). A partir del MNE se calculó el MAU y MAU\%, a fin de determinar cuáles son las unidades anatómicas con mayor representación. Finalmente, para evaluar las actividades de transporte, también se tomó en cuenta la abundancia de las distintas unidades anatómicas representadas en función de los índices de anatomía económica carne + médula calculados para este taxón (Borrero 1990).

Con respecto a la evaluación de las actividades relacionadas con la reducción de carcasas, se registró la presencia de huellas antrópicas (Mengoni Goñalons 1999; De Nigris 2004; Muñoz 2008), diferenciándolas de acuerdo con sus categorías (corte, raspado, negativo de impacto, entre otras), así como el tipo de fractura intencional (espiraladas, longitudinales o transversales), determinando si el hueso se encontraba en estado fresco (green) o no (no green) en el momento de su fractura (Mengoni Goñalons 1999; De Nigris 2004). 
Los análisis tafonómicos presentados aquí apuntan a conocer el grado de preservación y la integridad de las muestras, dado que en Tierra del Fuego actúan agentes no antrópicos y procesos que pudieron haber contribuido a la formación y transformación de los conjuntos óseos considerados (Borrero 1988, 1990, Mameli y Estévez Escalera 1999-2000; Estévez Escalera y Mameli 2000). Para ello, se evaluaron los estadios de meteorización de cada espécimen (Behrensmeyer 1978), la acción de carnívoros o roedores, las improntas de raíces y el grado de fragmentación (Borrero 1988; Mengoni Goñalons 1999).
En la Tablas 3.1 y 3.2 se presentan los resultados del análisis faunístico efectuado para los distintos conjuntos de Heshkaia. En el nivel inter-sitios, se observa variabilidad en cuanto a la abundancia de especímenes óseos de camélidos, así como en la representación de partes esqueletarias (Tabla 3.1 y 3.2). Asimismo, se presentan los estadios de meteorización (Figura 2), que sugieren una baja exposición de los conjuntos óseos. A continuación, se desarrollan los resultados alcanzados en el estudio de las muestras analizadas.

Tabla 3.1. NISP, MNE, MNI, MAU, MAU\% y NISP:MNE de los especímenes de guanacos en Heshkaia 28, 30, 34, 35 y 39.

\begin{tabular}{|c|c|c|c|c|c|c|c|c|c|c|c|c|c|c|c|c|c|c|c|c|c|c|c|c|}
\hline \multicolumn{2}{|c|}{$\begin{array}{r}\begin{array}{r}\text { Unidades } \\
\text { anatómicas }\end{array} \\
\text { Sitios }\end{array}$} & \multirow[t]{2}{*}{ 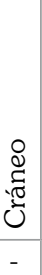 } & \multirow{2}{*}{ 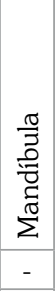 } & \multirow{2}{*}{$\begin{array}{l}\stackrel{n}{x} \\
-\end{array}$} & \multirow{2}{*}{$\begin{array}{l}\frac{a}{0} \\
\frac{0}{0} \\
\frac{0}{2} \\
0 \\
0 \\
-\end{array}$} & \multirow{2}{*}{ 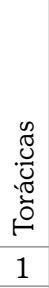 } & \multirow{2}{*}{ 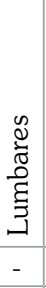 } & \multirow{2}{*}{$\begin{array}{l}\frac{0}{0} \\
\frac{\pi}{0} \\
\frac{\pi}{\pi} \\
-\end{array}$} & \multirow{2}{*}{ 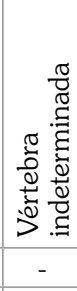 } & \multirow{2}{*}{ 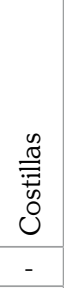 } & \multirow{2}{*}{ 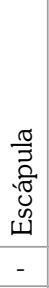 } & \multirow{2}{*}{$\begin{array}{l}\frac{n}{2} \\
\frac{n}{2} \\
-\end{array}$} & \multirow[t]{2}{*}{ 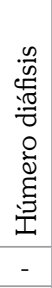 } & \multirow{2}{*}{ 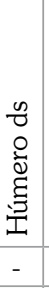 } & \multirow[t]{2}{*}{ 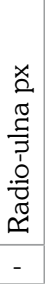 } & \multirow[t]{2}{*}{ 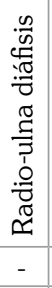 } & \multirow[t]{2}{*}{ 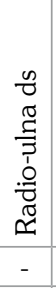 } & \multirow[t]{2}{*}{ 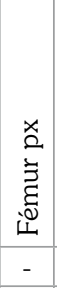 } & \multirow[t]{2}{*}{ 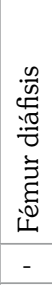 } & \multirow{2}{*}{ 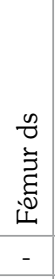 } & \multirow{2}{*}{\multicolumn{2}{|c|}{ 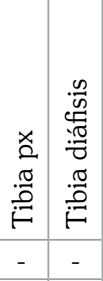 }} & $\begin{array}{l}0 \\
0 \\
.0 \\
0 \\
\stackrel{0}{0}\end{array}$ & \\
\hline & NISP & & & & & & & & & & & & & & & & & & & & & & & \\
\hline $\mathrm{N}$ & MNE & - & - & - & - & 1 & - & - & - & - & - & - & - & - & - & - & - & - & 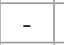 & - & - & - & - & \\
\hline ¿ & [NI & - & - & - & & 1 & - & - & 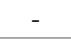 & 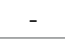 & - & - & - & - & - & - & - & - & - & - & 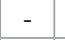 & - & - & \\
\hline 瑴 & IA & - & - & - & - & 0,1 & - & 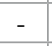 & - & - & - & - & - & - & - & - & - & - & 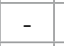 & - & 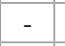 & - & - & \\
\hline $\bar{s}$ & $\mathrm{AA}$ & - & - & - & - & 0,5 & - & - & - & - & - & - & - & - & - & - & - & - & - & - & 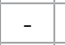 & - & - & \\
\hline & NIS & - & - & - & - & & & $\ldots$ & & $\ldots$ & 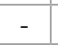 & - & 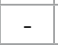 & & - & - & 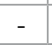 & - & & - & & - & & \\
\hline & & - & - & 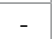 & - & & -5 & - & - & 1 & - & 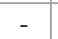 & - & - & 1 & - & - & - & 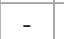 & - & 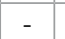 & - & & \\
\hline$m$ & I & - & - & - & - & & 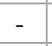 & - & - & & - & 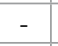 & - & - & 1 & - & - & - & 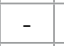 & - & - & - & - & \\
\hline & $\mathrm{MN}$ & -1 & 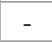 & 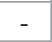 & - & & & - & - & & - & - & - & - & & - & - & - & - & - & - & - & & \\
\hline & $\mathrm{MAI}$ & - & - & - & - & & - & - & - & & 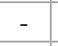 & 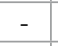 & - & - & 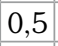 & - & - & - & 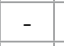 & - & - & - & - & \\
\hline & MAU & - & - & - & - & & - & 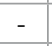 & - & & - & - & - & - & 100 & - & - & - & - & - & - & - & - & \\
\hline & NIS & - & - & - & - & $t$ & & - & - & & ( & - & - & - & 1 & - & - & - & - & - & - & - & & \\
\hline & & 11 & 3 & 2 & 17 & 5 & 15 & 2 & 12 & 20 & 3 & 5 & 3 & 2 & 1 & 1 & 3 & 3 & 4 & 1 & 1 & 1 & 1 & \\
\hline$\stackrel{\infty}{\ll}$ & MN & 1 & 1 & 2 & 6 & 2 & $\varepsilon$ & 2 & & & & & 1 & & & 1 & & 2 & 2 & & & 1 & & \\
\hline 安 & $=$ & & 1 & 2 & 1 & 1 & 1 & 1 & & & & 2 & 1 & 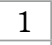 & 1 & 1 & & 1 & 1 & 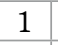 & 1 & 1 & & \\
\hline 羑 & MA & 1 & 1 & 2 & 2 & 0,2 & 1 , & & & & 1,5 & 1,5 & 0,5 & 0,5 & 0,5 & 0,5 & 1 & 1 & 1 & 5 & 0,5 & 0,5 & 5 & \\
\hline$\sqrt{5}$ & MAI & & 40 & & & 6,4 & & & & & & & 20 & 20 & 20 & 20 & 0 & 40 & 40 & 20 & 20 & 20 & 0 & \\
\hline & & & 3 & & & $\Omega_{0}$ & & & & & & & 1,5 & & & 1 &, 5 & 1,5 & & & & & & \\
\hline & & 5 & 2 & & & 5 & & & 9 & & & & & 2 & 4 & 1 & - & 2 & 3 & 6 & 9 & 4 & & \\
\hline & & & 2 & & & 3 & & - & 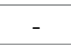 & & & & & 2 & 3 & 1 & - & 2 & 2 & & 5 & 1 & & \\
\hline & MN & & 2 & & 1 & 1 & 2 & - & & & & 1 & & 2 & 2 & 1 & - & - & 2 & 2 & 4 & 1 & & \\
\hline & MAU & 1 & 2 & & 0,6 & 0,3 & 0,7 & - & - & 4 & 0,5 & 0,5 & 0,5 & 1 & 1,5 & 0,5 & - & 1 & 1 & 2 & 2,5 & 0 & 1 & \\
\hline & MAU\% & 10 & 80 & & 24 & 10 & 28 & 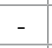 & - & 5,2 & 20 & 20 & 20 & 40 & 60 & 20 & - & 40 & 40 & 80 & 100 & 20 & 40 & \\
\hline & NISP: & 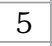 & 1 & & 1,7 & 1,7 & 4 & - & 2 & 3,6 & 1 & 3 & 4 & 1 & 1,3 & 1 & - & 1 & 1,5 & 1,5 & 1,8 & 4 & 1 & \\
\hline & NIS & 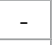 & $\Gamma_{-1}$ & - & - & - & 2 & - & - & 1 & & $-T_{2}$ & 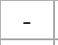 & - & 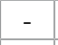 & - & - & - & - & - & - & - & - & \\
\hline 4 & MN & & 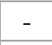 & $\begin{array}{lll}- & -\end{array}$ & - & - & . & - & - & & & - & - & - & - & - & - & - & - & - & - & - & - & \\
\hline$\frac{\pi}{4}$ & $\mathrm{M}$ & 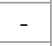 & - & 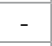 & - & - & & - & - & & & 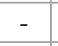 & 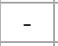 & - & - & - & - & - & - & - & - & - & - & \\
\hline 姜 & & - & - & 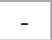 & - & & 0 & & - & & & - & - & - & - & - & - & - & 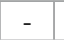 & - & & 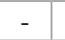 & & \\
\hline u & & - & - & - & - & - & 14 & - & & & & - & - & - & - & - & - & - & 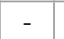 & - & - & - & - & \\
\hline & NISP:MN & - & - & - & - & - & & - & - & & & - & - & - & - & - & - & - & & & & & - & \\
\hline
\end{tabular}


Tabla 3.2. NISP, MNE, MNI, MAU, MAU\% y NISP:MNE de los especímenes de guanacos en Heshkaia 28, 30, 34, 35 y 39 (continuación tabla 3).

\begin{tabular}{|c|c|c|c|c|c|c|c|c|c|c|c|c|c|c|c|c|c|c|c|c|c|}
\hline \multicolumn{2}{|c|}{ Sitios } & 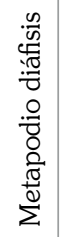 & $\begin{array}{l}0 \\
0 \\
0 \\
0 \\
0 \\
0.0 \\
0 \\
\sum \\
\sum \\
\Sigma\end{array}$ & 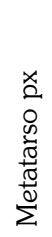 & $\begin{array}{l}0 \\
0 \\
0 \\
0 \\
0 \\
\pi \\
\pi \\
\Sigma\end{array}$ & 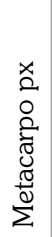 & 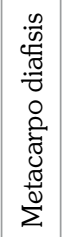 & 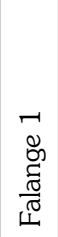 & 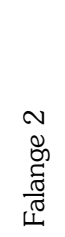 & $\begin{array}{l}m \\
0 \\
0 \\
0 \\
\frac{\tilde{\sigma}}{\widetilde{1}} \\
\omega\end{array}$ & 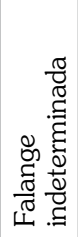 & 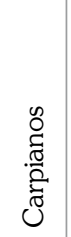 & 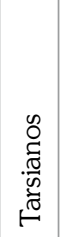 & 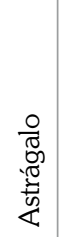 & 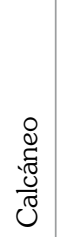 & 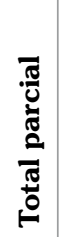 & 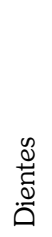 & 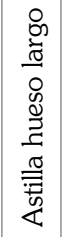 & 爰 & $\begin{array}{l}\text { త్ర } \\
\text { త్త }\end{array}$ & ڤేँّ \\
\hline \multirow{6}{*}{ 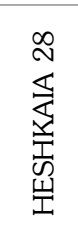 } & NISP & 1 & - & - & - & - & - & 4 & - & - & - & - & - & - & - & 6 & - & 3 & 2 & - & 11 \\
\hline & MNE & 1 & - & - & - & - & - & 3 & - & - & - & - & - & - & - & 5 & - & - & - & - & 5 \\
\hline & MNI & 1 & - & - & - & - & - & 1 & - & - & - & - & - & - & - & 1 & - & - & - & - & 1 \\
\hline & MAU & 0,3 & - & - & - & - & - & 0,5 & - & - & - & - & - & - & - & - & - & - & - & - & - \\
\hline & MAU\% & 50 & - & - & - & - & - & 100 & - & - & - & - & - & - & - & - & - & - & - & - & - \\
\hline & NISP:MNE & 1 & - & - & - & - & - & 1,3 & - & - & - & - & - & - & - & 1,2 & - & - & - & - & 2,2 \\
\hline \multirow{6}{*}{ 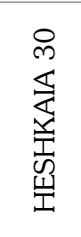 } & NISP & 1 & - & - & - & - & - & - & - & 2 & - & - & 2 & - & - & 14 & - & 5 & 6 & - & 25 \\
\hline & MNE & 1 & - & - & - & - & - & - & - & 2 & - & - & 2 & - & - & 12 & - & - & - & - & 12 \\
\hline & MNI & 1 & - & - & - & - & - & - & - & 1 & - & - & 1 & - & - & 1 & - & - & - & - & 1 \\
\hline & MAU & 0,3 & - & - & - & - & - & - & - & 0,3 & - & - & 0,2 & - & - & - & - & - & - & - & - \\
\hline & MAU\% & 50 & - & - & - & - & - & - & - & 50 & - & - & 32 & - & - & - & - & - & - & - & - \\
\hline & NISP:MNE & 1 & - & - & - & - & - & - & - & 1 & - & - & 1 & - & - & 1,2 & - & - & - & - & 2,1 \\
\hline \multirow{6}{*}{ 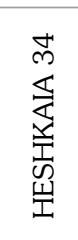 } & NISP & 14 & 5 & 1 & - & 2 & 1 & 24 & 6 & 6 & 11 & 12 & 9 & 1 & - & 211 & 6 & 104 & 282 & 10 & 613 \\
\hline & MNE & 5 & 2 & 1 & - & 1 & 1 & 9 & 4 & 6 & - & 12 & 9 & 1 & - & 102 & - & - & - & - & 102 \\
\hline & MNI & 3 & 2 & 1 & - & 2 & 1 & 2 & 1 & 1 & - & 2 & 1 & 1 & - & 4 & - & - & - & - & 4 \\
\hline & MAU & 2,5 & 1 & 0,5 & - & 0,5 & 0,5 & 1,3 & 0,5 & 0,8 & - & 0,9 & 0,8 & 0,5 & - & - & - & - & - & - & - \\
\hline & MAU\% & 100 & 40 & 20 & - & 20 & 20 & 50 & 20 & 30 & - & 34 & 30 & 20 & - & - & - & - & - & - & - \\
\hline & NISP:MNE & 2,8 & 2,5 & 1 & - & 2 & 2 & 2,7 & 1,5 & 1,2 & - & 1 & 1 & 1 & - & 2,2 & - & - & - & - & 6 \\
\hline \multirow{6}{*}{ 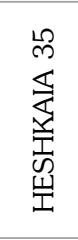 } & NISP & 7 & 4 & - & - & 1 & - & 22 & 17 & 4 & 1 & 26 & 18 & 1 & 6 & 226 & 8 & 165 & 428 & 7 & 834 \\
\hline & MNE & 4 & 3 & - & - & 1 & - & 6 & 9 & 4 & 1 & 25 & 18 & 1 & 4 & 124 & - & - & & - & 124 \\
\hline & MNI & 1 & 1 & - & - & 1 & - & 3 & 2 & 1 & 1 & 3 & 2 & 1 & 2 & 4 & - & - & & - & 4 \\
\hline & MAU & 2 & 1,5 & - & - & 0,5 & - & 0,8 & 1,1 & 0,5 & 0 & 1,8 & 1,6 & 0,5 & 2 & - & - & - & & - & - \\
\hline & MAU\% & 80 & 60 & - & - & 20 & - & 30 & 45,2 & 20 & 5 & 71,6 & 63 & 20 & 80 & - & - & - & - & - & - \\
\hline & NISP:MNE & 1,8 & 1,3 & - & - & 1 & - & 3,7 & 1,9 & 1 & 1 & 1,04 & 1 & 1 & 1,5 & 1,8 & & - & - & - & 6,7 \\
\hline \multirow{6}{*}{ 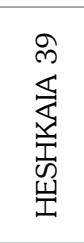 } & NISP & 1 & 4 & - & 1 & - & - & - & - & - & - & - & - & - & - & 9 & - & 6 & 6 & - & 21 \\
\hline & MNE & 1 & 2 & - & 1 & - & - & - & - & - & - & - & - & - & - & 6 & - & - & - & - & 6 \\
\hline & MNI & 1 & 2 & - & 1 & - & - & - & - & - & - & - & - & - & - & 2 & - & - & - & - & 2 \\
\hline & MAU & 0,5 & 1 & - & 0,5 & - & - & - & - & - & - & - & - & - & - & - & - & - & - & - & - \\
\hline & MAU\% & 50 & 100 & - & 50 & - & - & - & - & - & - & - & - & - & - & - & - & - & - & - & - \\
\hline & NISP:MNE & 1 & 2 & - & 1 & - & - & - & - & - & - & - & - & - & - & 1,5 & - & - & - & - & 3,5 \\
\hline
\end{tabular}

\section{RESULTADOS}

\section{Heshkaia 28}

Este sitio se ubica en el límite occidental de la localidad (54 56' 50,5” S - 66 50'34,2” O), por encima de un paleo-acantilado de escasa altura y actualmente en un ámbito de bosque. Cuenta con el menor número de especímenes óseos sobre el total de la muestra (ver Tabla 2). Esto se evidencia particularmente en los escasos restos de guanacos
(NISP=11) y en el MNI, que dio cuenta de solo un individuo.

Como consecuencia de los escasos especímenes, la diversidad de partes esqueletarias es muy baja, registrándose una mayor representación de elementos del esqueleto apendicular (ver Tablas 3.1 y 3.2). Este último muestra al metapodio y falange como la unidad anatómica más representada, a partir de la cuantificación en MAU y MAU\%.

Estas unidades anatómicas presentan un bajo índice de utilidad (Borrero 1990), por lo que tienen 


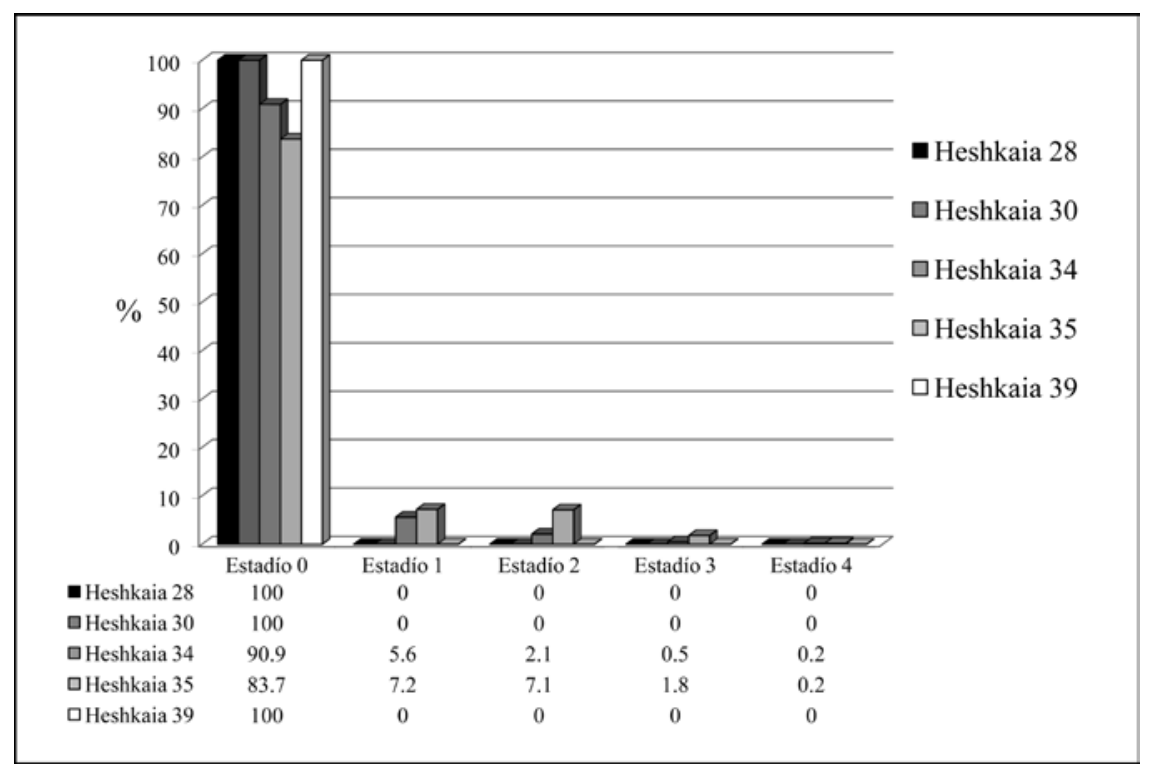

Fig. 2. Estadíos de meteorización en Heshkaia 28, 30, 34, 35 y 39.

menores posibilidades de ser acarreados desde el punto de encuentro al ámbito residencial. Generalmente, éstas llegan al lugar de consumo como parte del paquete transportable, compuesto por los miembros apendiculares con valores nutricionales más atractivos, como ser el fémur. No obstante, si bien los metapodios y falanges cuentan con poca carne, las marcas de corte y de fragmentación, como veremos, indican que la cavidad medular de estos elementos óseos ha sido efectivamente utilizada para el consumo.

Con relación al perfil etario, sólo se registraron dos especímenes fusionados correspondientes a la primera falange, lo cual indica la presencia de al menos un camélido con edad inferior a los 24 meses (Kaufmann 1999).

Respecto de la acción térmica, predominan los especímenes no termoalterados $(81,8 \%)$. Los restantes son ubicables en el estadio uno de termoalteración (Mengoni Goñalons 1999).

Las marcas antrópicas son de corte, y están representadas en el 55\% de los especímenes de guanacos. Se encuentran en astillas de huesos largos, falanges y metapodios. Las marcas de corte sobre estos dos últimos elementos están ubicadas cerca de las articulaciones, lo cual estaría relacionado con la remoción de los tejidos blandos asociados al hueso y con la desarticulación (ver Muñoz 2008).
En general, las marcas de corte registradas están ligadas con la morfología de fractura ósea y con el grado de fragmentación, rasgos que se relacionan con las actividades orientadas a la extracción de la médula. Como se puede observar (ver tablas 3.1 y 3.2), el índice de fragmentación (NISP: MNE) del conjunto total sugiere que los huesos se encuentran prácticamente íntegros, con una mayor incidencia en el grado de fragmentación correspondiente a las falanges. En este sentido, si bien no evidenciamos marcas de machacado o percusión, la mayor parte de las roturas se produjeron cuando el hueso aún estaba fresco (green), tal como lo evidencia la forma de las mismas (en espiral). Asimismo, se han observado pocos especímenes con fragmentación de carácter postdepositacional (con fracturas escalonadas). Es interesante señalar que parte de estas fracturas en estado fresco han sido observadas en falanges, las cuales tienen cavidades medulares menores en relación con elementos de mayores dimensiones (como el fémur y la tibia). Como mencionamos previamente, esto permitiría sugerir que la explotación de la médula -tanto de las grasas de mayor rendimiento como las más marginales- ha sido una práctica generalizada aún en aquellos huesos que poseen cavidades medulares muy reducidas.

En lo relacionado con las marcas no antrópicas, en este conjunto no han sido registradas sobre los 
restos de guanacos, exceptuando improntas de raíces sólo en un espécimen. Por último, la meteorización no afectó a los restos de camélidos (Ver Figura 2).

\section{Heshkaia 30}

Se encuentra al pie de un barranco de escasa altura y contiguo a una playa activa (54 $57^{\circ}$ 05,0" S - 66 49' 38,6" O). Registra un corte en toda su longitud, producto de erosión marina. El número de especímenes de guanacos es también reducido (NISP=25) y el MNI es de un individuo.

Aunque el NISP se mantiene bajo en este sitio y el MNI no difiere del sitio 28, se registra un aumento en la variabilidad de partes esqueletarias presentes en el conjunto, con una mayor representación de partes apendiculares (ver tablas 3.1 y 3.2). Al respecto, el radio-ulna es el elemento más representado por el MAU y MAU\%, seguido por tarsianos, falanges y metapodios.

Por otra parte, en aquellos casos en los cuales se pudo determinar el grado de fusión, se evidencia una mayor representación de especímenes no fusionados $(77,8 \%)$ por sobre los fusionados $(22,2 \%)$. Los primeros corresponden a epífisis y cuerpos sin fusionar de vértebras torácicas, así como también a falanges sin fusionar. Esto estaría indicando el ingreso al sitio de individuos juveniles con edades inferiores a los tres años (Kaufmann 2009).

Las marcas antrópicas aparecen en un $24 \%$ de los especímenes. Estas son marcas de corte, excepto un negativo de impacto producido sobre una astilla de hueso largo. Las marcas se distribuyen sobre restos axiales como apendiculares, aunque son más notables en estos últimos. Es interesante destacar la presencia de marcas de corte sobre tarsianos y vértebras torácicas, indicando posiblemente actividades de desarticulación y cuereo in situ (Muñoz 2008).

En lo que hace al índice de fragmentación (ver Tablas 3.1 y 3.2), al igual que en el sitio 28 los especímenes de camélidos se encuentran prácticamente completos. No obstante, cabe mencionar la presencia de fracturas en estado fresco evidenciadas en metapodios, radio-ulna y astillas de huesos largos, sugiriendo actividades relacionadas con la extracción de médula.

No se observan huellas producidas por agentes naturales y las improntas de raíces solo afectaron al $20 \%$ del conjunto. Respecto de la acción térmica, predominan los especímenes no termoalterados (81,8 \%); los restantes muestran el estadio uno de termoalteración (Mengoni Goñalons 1999).

En este conjunto no se registran efectos de la meteorización, ya que en su totalidad se observa el estadio cero (ver figura 2). Esto permite señalar un proceso de enterramiento homogéneo y una baja exposición aérea. Por lo tanto, esta situación, sumada a la nula presencia de marcas de roedores o carnívoros, permitiría sugerir que el conjunto tiene una alta integridad.

\section{Heshkaia 34}

Este es el sitio más extenso de la localidad Heshkaia (ver Tabla 1). Se ubica sobre un nivel de paleoplaya y al pie de un barranco (54 $57^{\circ} 13,9^{\prime}$ " S - 66 49' 02,1" O). Presenta un mayor número de especímenes de camélidos en comparación con los sitios ya descriptos (ver Tabla 2), lo cual se evidencia también con un aumento en el MNI a cuatro individuos.

En este sitio se encuentran presentes todas las unidades anatómicas, con una representación bastante proporcional del esqueleto axial y del esqueleto apendicular (ver Tablas 3.1 y 3.2). Esto se observa también al considerar la cuantificación por MAU y MAU\%, dado que las unidades anatómicas más abundantes del esqueleto axial son los axis, escápulas y pelvis, y del esqueleto apendicular son los metapodios y primeras falanges (ver Tablas 3.1 y 3.2). Por lo tanto, se destaca una alta diversidad de partes del esqueleto representadas, sin un patrón claro de selectividad en función de su rendimiento económico.

Los especímenes no fusionados $(57,4 \%)$ predominan sobre los fusionados $(42,6 \%)$, aunque los porcentajes son relativamente parejos. De los primeros, se trata de metapodios, falanges, radioulna, vértebras y un isquion suelto. Con relación al perfil de mortalidad, se observa un metapodio proximal sin fusionar, lo que indica la presencia de al menos un individuo cría con menos de seis meses (Kaufmann 2009). A su vez, el isquion no fusionado sugiere un individuo menor a los doce meses de vida. El resto de los elementos son de individuos con edades superiores al año, presentándose tanto animales juveniles como adultos. Semejante perfil etario sustentaría la idea de caza de grupos familiares 
y/o de tropillas de hembras, ya que a estos individuos no se los puede encontrar en otras agrupaciones (Kaufmann 2009).

Los especímenes termoalterados son escasos (8,6\%); en su mayoría se encuentran en estado quemado y, en menor medida, carbonizados.

El porcentaje de especímenes con marcas antrópicas es 16,3\%. Están representadas principalmente por marcas de corte, distribuidas tanto en el esqueleto axial como apendicular (siendo particularmente notables sobre las costillas) a partir de marcas de corte para el descarne y la desarticulación. También están presentes de manera importante en los procesos de vértebras cervicales y lumbares, lo que sugiere actividades de extracción de tejido blando. Las marcas de corte en el esqueleto apendicular se presentan mayormente sobre metapodios y falanges, y por su distribución estarían indicando actividades de desarticulación y cuereo (Muñoz 2008). Las marcas de percusión y machacamiento se presentan escasamente sobre un fragmento de radio-ulna y un metapodio, relacionadas con la extracción de médula ósea.

A diferencia de los conjuntos previamente descriptos, este sitio cuenta con un alto grado de fragmentación en los restos óseos de camélidos (94\%). El 24,6\% de las fracturas corresponden a roturas intencionales de huesos largos cuando estos aún se encontraban en estado fresco (Mengoni Goñalons 1999). Por lo tanto, si bien se observan escasas marcas de impacto sobre los huesos largos, tanto la morfología como el alto índice de fragmentación permitirían sugerir actividades antrópicas relacionadas con la extracción de la médula ósea, así como el procesamiento integral de las carcasas.

Las marcas no antrópicas son escasas, presentándose huellas de carnívoros y roedores sólo en seis especímenes sobre el total de la muestra de camélidos. Las improntas de radículas tampoco muestran una frecuente acción sobre la muestra, ya que sólo se observan en un 21,9\% de los especímenes. Esto estaría indicando que la conformación y acumulación del conjunto es absolutamente de origen antrópico, y que el reconocimiento de las distintas marcas culturales sobre los huesos no presentaría grandes obstáculos. Asimismo, la meteorización, al igual que en los demás sitios hasta ahora mencionados, tuvo una escasa incidencia en la conformación del conjunto (ver Figura 2).

\section{Heshkaia 35}

Se encuentra en un ámbito de bosque (5456'58,9"S - 6649'22,5”O) (ver Tabla 1) y cuenta con la mayor abundancia de especímenes óseos de camélidos del total de los sitios tratados en este informe (ver Tablas 3.1 y 3.2); cabe señalar que es el que cuenta con mayor superficie excavada hasta el momento. El NISP de guanacos es de 180 especímenes provenientes del sondeo más 654 resultantes de la excavación. El MNI dio cuenta de cuatro individuos.

En relación con la diversidad anatómica, el MAU y el MAU\% muestran a la tibia como la unidad más representada, seguida por la mandíbula, el fémur, el metapodio y el calcáneo (ver Tablas 3.1 y 3.2). Si bien la representación del esqueleto apendicular $(79,8 \%)$ supera la del esqueleto axial $(20,2 \%)$, todas las unidades anatómicas se encuentran presentes. A esta situación se agrega la llamativa abundancia de metapodios, falanges, calcáneos, carpianos y tarsianos en la muestra, los cuales poseen escaso rendimiento económico para la dieta humana (Borrero 1990). Esta variabilidad de elementos óseos -con contrastable índice de utilidad- estaría indicando que las carcasas de guanacos habrían ingresado completas al sitio, aunque con una preferible selección de las extremidades.

Los especímenes no fusionados son 41 . En relación a las clases de edad, las falanges estarían indicando la presencia de individuos menores a los 24 meses de edad. El resto de los elementos indican individuos con edades superiores.

Las alteraciones térmicas se destacan en este sitio dado su alto grado de variabilidad. Por un lado, en la totalidad de los especímenes provenientes del sondeo realizado en el conchero, con la excepción de tres huesos quemados, no percibe ningún sometimiento al calor. En contraste, el conjunto por fuera del montículo evidencia una alta incidencia térmica, con el 25,7\% de los especimenes en estado quemado, el 26,9\% carbonizado, y el 14,5\% calcinado. Esta alta presencia de huesos sometidos a elevadas temperaturas dio lugar a que ciertos procesos antrópicos no pudieran ser medidos a partir de esta variable, dado que los huesos carbonizados y calcinados están más relacionados con el descarte de piezas óseas en los fogones o con su uso como material combustible (Mengoni Goñalons 1999). Por esta razón, su valor 
informativo acerca de las actividades estrictamente culinarias es limitado.

En el 16\% de los especímenes se encuentran marcas antrópicas. La casi totalidad de las marcas son de corte, exceptuando nueve casos que poseen marcas de percusión y cuatro de machacado. Estas marcas se encuentran distribuidas en todas las regiones del esqueleto, principalmente sobre costillas y falanges, y sugieren actividades de descarne y desarticulación en el sitio (Muñoz 2008). Asimismo, las marcas de percusión están presentes en gran medida sobre astillas de hueso largo y diáfisis de tibia y fémur, indicando tareas de extracción de la médula ósea contenida en estas osamentas.

La intensidad de la fragmentación es de 90\% y los especímenes enteros corresponden a elementos compactos o de pequeño tamaño (carpianos, tarsianos, sesamoideos y falanges). La relación NISP:MNE indica que las unidades anatómicas del esqueleto axial más fragmentadas son el cráneo, vértebras cervicales y costillas, mientras que en el segmento apendicular las razones más elevadas corresponden al fémur, húmero, metapodios y falanges (ver Tablas 3.1 y 3.2). El 44\% de las fracturas en las extremidades se produjeron cuando el hueso estaba en estado fresco.

$\mathrm{Al}$ igual que en los demás sitios, se registra poca acción de carnívoros y roedores. Los primeros se observan en siete especímenes y los segundos sólo en dos. Lo mismo puede decirse en relación con las improntas de radículas, las cuales se hallan sólo sobre el 10,9\% de los restos de camélidos. Estos factores permiten sugerir que los agentes naturales no alteraron de manera intensiva al conjunto, facilitando la visibilidad de marcas antrópicas sobre los huesos.

Finalmente, como en todos los conjuntos, los especímenes óseos presentan una buena preservación general, y en su mayoría se encuentran en el estadio cero (ver Figura 2). Por lo tanto, esto indicaría que hubo una reducida exposición de los huesos.

\section{Heshkaia 39}

Es un sitio de escasa extensión y profundidad que se encuentra en un ámbito de bosque

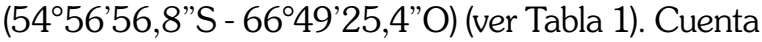
con pocos especímenes en general, aunque predominan los camélidos. El MNI es de dos individuos.
Si bien los metapodios son los huesos más bundantes en el conjunto, la presencia de vértebras lumbares y costillas indica que el esqueleto axial también fue acarreado al sitio (ver Tablas 3.1 y 3.2). Dado el bajo índice de utilidad de carne de los metapodios, su predominante abundancia podría sugerir actividades orientadas a la extracción de la médula ósea. Como veremos, este hecho quedaría sustentado con la morfología de las fracturas.

En cuanto al perfil etario, se observan tres especímenes de metapodios con la epífisis distal sin fusionar, y una epífisis proximal de metatarso fusionada. El primer caso, indicaría la presencia de individuos juveniles con edades entre 24 y 36 meses, mientras que en el segundo se sugiere una cría menor a seis meses (Kaufmann 2009). Este perfil de mortalidad permitiría suponer, al igual que en el sitio 34, la caza humana sobre grupos familiares y/o tropillas de hembras y crías.

$\mathrm{Al}$ igual que en los demás conjuntos, los especímenes termoalterados son escasos. En este sitio sólo se cuenta con la presencia de tres especímenes quemados (14,3\%).

Las marcas antrópicas se encuentran sobre el 19\% de los huesos de camélidos. En su mayoría son de corte, aunque también se observan negativos de impacto y marcas de machacado. Estos rastros se presentan sobre metapodios y astillas de hueso largo, implicando el desarrollo de actividades de extracción de médula, así como de tejido blando (Muñoz 2008).

Finalmente, los factores naturales no alteraron al conjunto, aunque cabe mencionar la presencia de manganeso sobre el 76,2\% de los restos de camélidos. Lo mismo puede decirse del estadio de meteorización (cero sobre todos los restos), lo que indica alta integridad y baja exposición del conjunto.

\section{DISCUSIÓN}

Los resultados de los cinco conjuntos analizados muestran que los guanacos fueron transportados y procesados en todos los sitios excavados. Con independencia del tamaño de los conjuntos, los individuos son siempre pocos y no muestran diferencias significativas en el número de animales representados. En los sitios 28 y 30 el MNI dio cuenta de sólo un individuo, mientras que en el 39 se observan dos. Estos conjuntos tienen una muestra 
muy reducida, por lo que la discusión relacionada con el procesamiento de guanacos en estos sitios es limitada y no será considerada en la discusión de este trabajo. A diferencia de estos contextos, en los sitios 34 y 35 casi todas las unidades anatómicas del guanaco se encuentran presentes (ver Tablas 3.1 y 3.2). A esta situación se suman números de especímenes más elevados, lo que posibilitan un mayor alcance en cuanto al grado de precisión en las interpretaciones, así como en las comparaciones a nivel inter-sitio.

En Heshkaia 34 la representación de las unidades anatómicas para el esqueleto axial y apendicular es bastante proporcional, pero con cierta variabilidad en cuanto a los elementos presentes en cada una de esas zonas (ver Tablas 3.1 y 3.2). Esta situación estaría indicando el transporte completo de guanacos sin selección de partes anatómicas en función de su rendimiento económico.

Respecto al procesamiento, las marcas de corte están presentes en todas las unidades anatómicas, indicando el aprovechamiento de distintas partes esqueletarias para el consumo. La información de las marcas culturales indica la presencia de actividades relacionadas con la desarticulación, la remoción de tejidos blandos asociados a los huesos y el procesamiento de estos últimos en sí in situ.

A la situación descripta en el párrafo anterior se suma el hecho de que los huesos se encuentran altamente fragmentados (94\%), y pocas partes esqueletarias aparecen completas. Esto es reflejo también de la mayor abundancia de especímenes que no pudieron ser asignados anatómicamente y del predominio de un alto índice de fragmentación. Asimismo, cabe mencionar que la mayor parte de las roturas se produjeron cuando los huesos se encontraban aún frescos, tal como lo evidencia la forma de las mismas (espiral y longitudinal). A su vez, se han observado pocos especímenes con fragmentación de carácter postdepositacional (como fracturas escalonadas, bordes astillados, etc.). Los huesos más fragmentados son los que poseen cavidad medular $\mathrm{u}$ órganos ricos en grasas. Se destaca también la significativa fragmentación de las falanges - primeras y segundas-, tanto en individuos adultos como en juveniles, pese a su bajo rendimiento nutricional (Borrero 1990). Podríamos sugerir entonces, que las huellas culturales y de fracturas estarían indicando un aprovechamiento integral y de actividades rela- cionadas con todos los estadios de procesamiento de las carcasas.

Heshkaia 35 plantea una situación comparativamente similar al sitio 34 en cuanto al manejo de las carcasas de guanacos. Como ya vimos, la cantidad de individuos representados, el análisis de la composición de los perfiles esqueletarios y su relación con la anatomía económica sugieren que el transporte selectivo en este sitio tampoco habría jugado un rol decisivo en la conformación de los perfiles anatómicos. En este mismo sentido, la configuración anatómica estaría reflejando los momentos finales de ese proceso más que los que tienen lugar al principio de la manipulación de las carcasas (Mengoni Goñalons 1999).

En cuanto al procesamiento y consumo, como mencionamos en el párrafo anterior, las evidencias para Heshkaia 35 permitirían sustentar que fue un lugar de consumo final de las presas. Esto se debe principalmente a que el conjunto cuenta con una gran diversidad de partes esqueletarias, tanto de la región axial como apendicular. A su vez, el alto índice de fragmentación (90\%) es congruente con la desorganización anatómica esperable en este tipo de sitios, así como la frecuencia de especímenes con huellas de procesamiento ya descriptas. Por último, la elevada cantidad de huesos fracturados intencionalmente es compatible con la rotura y segmentación de los huesos para la extracción y el consumo de médula ósea.

Con lo dicho hasta el momento, los datos relacionados con la obtención, transporte, procesamiento y consumo de guanacos permiten caracterizar su modalidad de aprovechamiento en Heshkaia 34 y 35. Por un lado, exceptuando la presencia de una cría menor a los seis meses en el sitio 34, la predación humana se habría focalizado en la explotación de animales mayores al año de edad. Por otro lado, la amplia diversidad de partes esqueletarias, el grado de desorganización anatómica, la frecuencia de especímenes con marcas de procesamiento y la cantidad de huesos fracturados intencionalmente, permitirían postular que no hubo evidencia de transporte selectivo desde los sitios de obtención hasta los lugares de procesamiento en ninguno de los dos conjuntos, reflejando el procesamiento y consumo final de ungulados in situ. Semejante escenario sugeriría proximidad entre el lugar de encuentro de las presas y la residencia, 
lo que habría facilitado el transporte completo de las mismas al campamento, para ser finalmente procesadas, consumidas y descartadas en el sitio.

Por último, en todos los conjuntos la meteorización y otros factores naturales no parecen haber influido de manera determinante en los restos faunísticos analizados aquí. Por lo tanto, se puede sostener que los agentes tafonómicos no modificaron sustancialmente la acumulación antrópica de los conjuntos, por lo que se evidencia una tendencia hacia una alta integridad del registro.

\section{CONSIDERACIONES FINALES}

En términos generales para los sitios excavados, los resultados sugieren que los grupos cazadoresrecolectores que ocuparon los sitios aquí analizados no seleccionaron las partes esqueletarias de guanacos en función de su valor nutricional. Los costos de transporte no habrían sido altos, requiriendo de un trozamiento inicial mínimo, no selectivo, orientado a dividir las carcasas en paquetes transportables. Estos aspectos permitirían sugerir cercanía de la localidad de matanza con la del procesamiento y consumo final. Esta situación de proximidad pudo haber estado influenciada por las características geográficas propias ya mencionadas para Moat.

Finalmente, es posible sugerir a modo de hipótesis que los cazadores-recolectores que habitaron Heshkaia pudieron haberse desplazado en rangos espaciales poco extensos para el aprovisionamiento de guanacos hacia lugares relativamente cercanos a los sitios costeros (daily foraging trips, sensu Binford 1980). Esto deberá ser analizado con mayor profundidad en el futuro, a partir de nuevos análisis zooarqueológicos y de la evaluación de otras líneas de evidencia que permitirán comprender mejor la naturaleza del registro.

\section{AGRADECIMIENTOS}

Estas investigaciones fueron realizadas con un subsidio de la Agencia Nacional para la Promoción Científica y Técnica (PICT 2010-1322). Agradecemos la lectura y las sugerencias de Luis A. Orquera sobre una versión preliminar de este trabajo. A María Paz Martinoli, María José Saletta, Angélica Tivoli y a Ernesto Piana por sus colaboraciones en los trabajos de campo y opiniones sobre este trabajo. Agrade- cemos asimismo a los evaluadores de este informe por sus lecturas críticas y sugerencias.

\section{BIBLIOGRAFÍA}

BEHRENSMEYER, A. K. 1978. Taphonomic and ecologic information from bone weathering. Paleobiology 4(2): 150-162.

BINFORD, L. 1980. Willow smoke and dogs' tails: hunter-gatherer settlement systems and archaeological site formation. American Antiquity 45(1): 4-20.

BONINO, N y FERNÁNDEZ, E. 1994. Distribución general y abundancia relativa de guanacos (Lama guanicoe) en diferentes ambientes de Tierra del Fuego, Argentina. Ecología Austral, 4:79-85.

BONINO, N. y PELLIZA SBRILLER, A. 1991a. Comparación de las dietas del guanaco, ovino y bovino en Tierra del Fuego, Argentina. Turrialba, 41 (4): 452-457.

1991b. Composición botánica de la dieta del guanaco (Lama guanicoe) en dos ambientes contrastantes de Tierra del Fuego, Argentina. Ecología Austral 1: 97-102.

ESTÉVEZ ESCALERA, J. y MAMELI, L. 2000. Muerte en el canal: experiencias bioestratigráficas controladas sobre la acción sustractiva de cánidos. Archaeofauna 9: 7-16.

BORRERO, L.A. 1988. Tafonomía Regional. En: De procesos, Contextos y Otros Huesos. Ratto, N y Haber, A. editores, pp. 9 - 15. Instituto de Ciencias Antropológicas, Universidad de Buenos Aires.

1990. Taphonomy of guanaco bones in Tierra del Fuego. Quaternary Research 34 (3): 361-371.

1990. Fuego-Patagonian bone assemblages and the problem of communal guanaco hunting. En: Hunters of the Recent Past. Davis, L. B. y Reeves, B. O. K. editores, 373-299. Unwin Hyman. Londres.

DE NIGRIS, M. 2004. El consumo en grupos cazadores recolectores: un ejemplo zooarqueológico de Patagonia Meridional. Sociedad Argentina de Antropología, Buenos Aires.

FITZ-ROY, R. 1839. Proceedings of the second expedition (1831-1836) under the command of captain Robert Fitz-Roy (R.N.). En: Narrative of the surveying voyages of his majesty's ships Adventure and Beagle between the years 1826 and 1836, Vol. II, 695 pp. Henry Colburn. Londres.

FRANGI, J.L. y RICHTER, L. L. 1994. Balances hidrícos de bosques de Nothofagus de Tierra del Fuego (Argentina). Revista de la Facultad de Agronomía 70: 65-79.

HEANE, E.; CAVAGNARO, F.P; ZANGRANDO, A.F. y CROJETOVICH, A.D. 2011. Relevamiento arqueológico. 
En: Relevamiento de la Estancia Moat, Provincia de Tierra del Fuego, Antártida e Islas del Atlántico Sur (República Argentina). Programa refugios de la Vida Silvestre. Tierra del Fuego, Ushuaia.

KAUFMANN, C, A. 2009. Estructura de edad y sexo en guanaco: estudio actualístico y arqueológico en Pampa y Patagonia. Sociedad Argentina de Antropología, Buenos Aires.

LANATA, J. L. 1990. Humans and terrestrial and sea mammals at Peninsula Mitre, Tierra del Fuego. En: Hunters of the recent past: 400-406. Davis, L. B. y B. O. K. Reeves editores. Unwin Hyman. Londres.

MAMELI, L. y ESTÉVEZ ESCALERA, J. 1999-2000. Seguimiento tafonómico-arqueológico sobre carcasas de camélidos, ovinos y bovinos en Tierra del Fuego (Argentina). Xama 12-14: 87-106.

MARTIAL, L.F. 1888. Historie du voyage. Mission Scientifique du Cap Horn (1882-1883), vol I. Gauthier-Villars. París.

MENGONI GOÑALONS, G. 1999. Cazadores de guanacos de la estepa patagónica. Sociedad Argentina de Antropología, Buenos Aires.

MONTES, C., D.; DE LAMO, A. y ZAVATTI, J. 2000. Distribución de abundancias de guanacos (Lama guanicoe) en los distintos ambientes de Tierra del Fuego, Argentina. Mastozoología Neotropical 7(1): 23-31.

MOORE, D. 1983. Flora of Tierra del Fuego. Anthony Nelson. Shropshire.

MUÑOZ, S. A. 2008. El procesamiento de los camélidos fueguinos en el pasado. Aspectos metodológicos y resultados alcanzados para el sector atlántico de Tierra del Fuego. En: Temas de Arqueología: Estudios tafonómicos y zooarqueológicos (I):77-97. Acosta, A.; Loponte, D.; Mucciolo, L. (compiladores). Instituto Nacional de Antropología y Pensamiento Latinoamericano, Buenos Aires.

ORQUERA, L. A. y PIANA, E. L. 1999a. Arqueología de la región del canal Beagle (Tierra del Fuego, República Argentina). Sociedad Argentina de Antropología, Buenos Aires.
ORQUERA, L. A. y PIANA, E. L. 1999b. La vida material y social de los Yámana. Editorial Universitaria de Buenos Aires, Buenos Aires.

RAEDEKE, K. 1976. El guanaco de Magallanes, Chile: Distribución y biología. Publicacion Tecnica-Corporacion Nacional Forestal. Santiago.

ROIG, C. y COLLADO, L. 2004. Antecedentes sobre turberas en Tierra del Fuego. En: Los Turbales de la Patagonia Bases para su inventario y la conservación de su biodiversidad. Blanco, D. y De la Balze, M.V. editores, 33-44. Wetlands International Publicación № 19, Buenos Aires.

SCHIAVINI, A.; ESCOBAR, J. y DEFERRARI, G. 2010. Cambios en la distribución estacional del guanaco en Tierra del Fuego. IV Reunión Binacional de Ecología. Facultad de Ciencias Exactas y Naturales de la Universidad de Buenos Aires. Argentina, Buenos Aires.

SCHLATTER, R. 2004. Fauna de turberas de la XII Región y Tierra del Fuego. En: Los Turbales de la Patagonia: Bases para su inventario y la conservación de su biodiversidad. Publicación No. 19. . Blanco, D. E. y V. M. de la Balze editores, Wetlands International. Argentina, Buenos Aires.

ZANGRANDO, A. F. 2010. Coastal Archaeology and HunterGatherers in Southeastern Tierra del Fuego. The Journal of Island and Coastal Archaeology 5(2): 288-291.

ZANGRANDO, A. F.; ALUNNI, D.; MARTINOLI, M. P.; TIVOLI A. y PIANA, E. 2010. Arqueología de la región de Moat (Tierra del Fuego, Argentina): estudios preliminares en la localidad arqueológica Heshkaia. Bárcena, J. R. y H. Chiavazza (eds.). Arqueología Argentina en el Bicentenario de la Revolución de Mayo, Actas XVII Congreso Nacional de Arqueología Argentina: 20052010. Argentina, Mendoza.

ZANGRANDO, A, F.; TIVOLI, A.; MARTINOLI, M.P. y ALUNNI, D. 2011. Heshkaia 35: nuevos datos sobre la arqueología de Moat (Tierra del Fuego). VIII Jornadas de Arqueología de la Patagonia. Argentina, Mendoza. 
